\title{
Necesidad de tratamiento ortodóncico en adolescentes de 12 años, ciudad de Diego de Almagro, Región de Atacama, Chile.
}

\section{Orthodontic treatment need in 12-year- old adolescents, city of Diego de Almagro, Atacama Region, Chile.}

\author{
Valentina Pedreros ${ }^{1}$, Gonzalo Peigna ${ }^{2}$, Luis González ${ }^{3}$, Antonieta Pérez-Flores ${ }^{4 *}$
}

\author{
1. Cirujana Dentista EDF, Servicio de Salud \\ Valdivia, Chile. \\ 2. Cirujano Dentista Consultorio Miraflores, Servicio \\ de Salud Araucanía Sur, Chile. \\ 3. Asesor Departamento de Salud Pública, \\ Secretaría Regional Ministerial de Salud Atacama, \\ Chile. \\ 4. Profesora Asociada Facultad de Odontología, \\ Universidad de Concepción, Chile. \\ * Correspondencia Autor: Antonieta Pérez- Flores \\ | E-mail: mperezf@gmail.com | Teléfono +569 \\ 98838301 \\ Trabajo recibido el 31/03/2020. \\ Aprobado para su publicación el 31/05/2020
}

\section{RESUMEN}

Objetivo: Determinar la necesidad de tratamiento ortodóncico en adolescentes de 12 años de Diego de Almagro, Chile, según el Índice de Estética Dental (IED). Materiales y Métodos: estudio descriptivo, observacional, transversal y no probabilístico. Se aplicó el IED en 97 estudiantes de 12 años de la localidad Diego de Almagro con un examen clínico estandarizado por una investigadora previamente calibrada, respetando las recomendaciones de la Organización Mundial de la Salud. La muestra fue de tipo censal. Se analizaron los criterios IED descriptivamente. Resultados: De 118 adolescentes, 97 reunían los criterios de inclusión, $17.52 \%$ obtuvo puntajes del IED $\leq 25$, indicativo de oclusión normal o maloclusión mínima, lo que determina tratamiento innecesario o poco necesario; $16.49 \%$ obtuvo una puntuación entre 26 y 30 , maloclusión manifiesta y una necesidad de tratamiento optativa; $16.49 \%$ obtuvo una puntuación entre 30 y 35 , maloclusión severa y una necesidad de tratamiento deseable por el paciente; $49.48 \%$ presentó un puntaje de IED $\geq 36$, indicativo de maloclusión muy severa o discapacitante con una necesidad de tratamiento obligatoria. Conclusiones: Existe una alta necesidad de tratamiento ortodóncico obligatorio en la población adolescente de 12 años, según el IED en que el $65.97 \%$ presentaba una maloclusión severa o muy severa.

PALABRAS CLAVE

Índice de estética dental; Prevalencia; Adolescentes; Tratamiento ortodóncico; Maloclusión; Necesidad de tratamiento.

Int. J. Inter. Dent Vol. 13(3); 127-131, 2020.

\section{ABSTRACT}

Objective: To determine the orthodontic treatment need in 12-year-old adolescents from the city of Diego de Almagro, Chile, according to the Dental Aesthetics Index (DAI) Materials and Methods: descriptive, observational, cross-sectional and nonprobabilistic study. The DAI was applied to 9712 -year-old students belonging to the city of Diego de Almagro, Atacama Region. The sample was of census type. The information was obtained through a standardized clinical examination performed by a previously calibrated researcher, fulfilling the recommendations described by the World Health Organization for this type of studies. The DAl criteria were analyzed descriptively. Results: 97 out of 118 students met the inclusion criteria, 45 (46.3\%) were males and $52(53.7 \%)$ females; $17.52 \%$ of the children examined obtained DAI scores $\leq 25$, indicating a normal occlusion or minimal malocclusion, which determines that the treatment is unnecessary or not necessary; $16.49 \%$ obtained a score between 26 and 30 , indicating a manifest malocclusion and an optional treatment need; $16.49 \%$ obtained a score between 30 and 35 with severe malocclusion and a treatment need desirable by the patient; $49.48 \%$ had a DAI score $\geq 36$, indicating a very severe or disabling malocclusion with a compulsory treatment need. Conclusions: There is a high mandatory orthodontic treatment need in the 12-year-old adolescent population, based on the fact that $65.97 \%$ of the studied sample through DAI presented a severe or very severe malocclusion.

\section{KEY WORDS}

Index of dental aesthetics; Adolescents; Orthodontic treatment.

Int. J. Inter. Dent Vol. 13(3); 127-131, 2020. 


\section{INTRODUCCIÓN}

En el sistema público de salud chileno, la ortodoncia es la especialidad odontológica que acumula el segundo mayor número de usuarios en la lista de espera después de la rehabilitación oral $^{(1)}$. El tratamiento de las maloclusiones, anomalías relacionadas con el crecimiento y desarrollo de los huesos maxilares durante la niñez y adolescencia, compromete la estética facial y las funciones masticatoria y fonética. Además, sus efectos incluyen esferas psicosociales, generando un impacto negativo en el diario vivir ${ }^{(2,3)}$.

Frente a una alta demanda por tratamientos ortodóncicos, se hace necesario priorizar utilizando parámetros estandarizados. Para esto se han desarrollado múltiples índices que han sido validados para medir la prevalencia y severidad de las maloclusiones junto con la necesidad de tratamiento ortodóncico ${ }^{(4,5)}$, siendo el Índice de Estética Dental (IED) el más utilizado a nivel mundial y el recomendado por la Organización Mundial de la Salud( ${ }^{(6)}$. El IED combina factores estéticos y funcionales, estableciendo un puntaje umbral a la necesidad de tratamiento ortodóncico, lo que ayuda a priorizar cuando la oferta de servicios dentales de especialidad es escasa(7).

El sistema de salud es en sí mismo un Determinante Social de la Salud (DSS), que se relaciona recíprocamente con el efecto de los otros $^{(8)}$.

El objetivo de esta investigación es conocer la prevalencia y severidad de las maloclusiones en escolares de 12 años de una localidad de la zona norte de Chile, para determinar la necesidad de tratamiento de ortodoncia de esta población y aportar en la organización de los servicios odontológicos locales de ortodoncia, fundamentado en el principio de equidad en el acceso a los servicios de salud.

\section{MATERIALES Y MÉTODOS}

Se realizó un estudio observacional descriptivo de corte transversal en escolares de 12 años de la localidad de Diego de Almagro, en la región de Atacama, Chile. Los criterios de inclusión fueron: pacientes con 12 años de edad cumplidos al 31 de marzo de 2017, que pertenezcan a los establecimientos educacionales de Diego de Almagro y aquellos que tengan el consentimiento informado firmado y aceptado por los padres y/o apoderados, junto al asentimiento de los adolescentes. Los criterios de exclusión fueron: pacientes con tratamiento previo o actual ortopédico/ortodóncico y pacientes con paladar hendido o fisurado, síndromes o enfermedades sistémicas incapacitantes.

Los procedimientos metodológicos estuvieron conforme a las normas éticas del comité de Bioética de la Facultad de Odontología de la Universidad de Concepción, Certificado $N^{\circ} 030 / 17$, y de acuerdo con la Asociación Médica Mundial y la Declaración de Helsinki (versión 2008).

De un total de 118 adolescentes, 97 cumplieron con los criterios de inclusión, el tamaño de la muestra fue de tipo censal.

Bajo la autorización de las instituciones, se entregó un consentimiento informado a cada apoderado, además se solicitó el consentimiento para obtener información socioeconómica del alumno.

Se realizó un examen clínico de la cavidad oral de los escolares seleccionados por una odontóloga calificada, quien realizó una calibración intraexaminador revisando modelos de yeso de pacientes no pertenecientes al estudio ${ }^{(4,5)}$, en dos oportunidades con una semana de intervalo, logrando un valor kappa intraexaminador de 0.7 .

El examen clínico se efectuó en los establecimientos educacionales, en una sala reservada, con el paciente sentado en una silla y el operador situado detrás de él, bajo luz artificial portátil (mediante linterna Energizer manos libres LED). Se utilizó guantes de látex, mascarillas y espejo intraoral $n^{\circ} 5$. Además, sonda Carolina del Norte para medir overjet y diastema interincisivo; y regla flexible para medir apiñamiento y espaciamiento dentario.

El ingreso de la información recopilada mediante fichas durante el examen clínico fue realizado por el examinador, datos que posteriormente fueron traspasados a una ficha electrónica realizada en google docs mediante un formulario ad-hoc.

Las variables estudiadas fueron sexo, pertenencia étnica y severidad de la maloclusión.

La obtención de la información se realizó utilizando el IED, índice que está dado por una ecuación de regresión estándar que incluye 10 componentes con sus correspondientes coeficientes (Tabla 1).

Los pacientes fueron analizados en cada componente del IED, los que a su vez fueron multiplicados por su correspondiente coeficiente de regresión. Los productos obtenidos se sumaron entre sí más la constante, obteniéndose el puntaje IED final. De esta forma, el IED es igual a (dientes visibles ausentes $\times 6)+($ apiñamiento $\times 1)+$
Tabla 1: IED, dado por ecuación de regresión estándar con sus 10 componentes y sus correspondientes coeficientes.

\begin{tabular}{|c|c|c|c|}
\hline $\mathbf{N}^{\circ}$ & Características & Coeficiente & $\begin{array}{c}\text { Peso } \\
\text { aproximado }\end{array}$ \\
\hline 1 & $\begin{array}{l}\text { Número de dientes visibles } \\
\text { ausentes (incisivos, caninos, } \\
\text { y premolares) }\end{array}$ & 5,76 & 6 \\
\hline 2 & $\begin{array}{l}\text { Evaluación de apiñamiento en } \\
\text { los segmentos incisales: } 0= \\
\text { no hay segmentos apiñados, } \\
1=\text { un segmento apiñado y } 2 \\
=\text { dos segmentos apiñados }\end{array}$ & 1,15 & 1 \\
\hline 3 & $\begin{array}{l}\text { Evaluación de espaciamiento } \\
\text { en los segmentos incisales: } \\
0=\text { no hay segmentos } \\
\text { espaciados, } 1=\text { un segmento } \\
\text { espaciado y } 2=\text { dos } \\
\text { segmentos espaciados }\end{array}$ & 1,31 & 1 \\
\hline 4 & $\begin{array}{l}\text { Diastema en la línea media } \\
(\mathrm{mm})\end{array}$ & 3,13 & 3 \\
\hline 5 & $\begin{array}{l}\text { Irregularidad anterior en el } \\
\text { arco superior }(\mathrm{mm})\end{array}$ & 6,75 & 7 \\
\hline 6 & $\begin{array}{l}\text { Irregularidad anterior en el } \\
\text { arco inferior }(\mathrm{mm})\end{array}$ & 1,34 & 1 \\
\hline 7 & Overjet maxilar $(\mathrm{mm})$ & 1,62 & 2 \\
\hline 8 & $\begin{array}{l}\text { Overjet mandibular (overjet } \\
\text { opuesto) }(\mathrm{mm})\end{array}$ & 3,68 & 4 \\
\hline 9 & Mordida abierta anterior (mm) & 3,69 & 4 \\
\hline 10 & $\begin{array}{l}\text { Relación molar antero- } \\
\text { posterior }(0=\text { normal y } \\
\text { Clase I de Angle, } 1=\text { Clase } \\
\text { II de Angle y } 2=\text { Clase III de } \\
\text { Angle) }\end{array}$ & 2,69 & 3 \\
\hline & Constante & 13,36 & 13 \\
\hline
\end{tabular}

*IED: Indice de Estética Dental

Tabla 2: Clasificación de maloclusión y necesidad de tratamiento según IED.

Clasificación

Calificación

Interpretación

Oclusión normal o Resultado del IED, Sin necesidad de maloclusión mínima menor o igual a 25 tratamiento o sólo un

$\begin{array}{lll}\text { Maloclusión definida } & \begin{array}{l}\text { Resultado del IED } \\ \text { entre } 26-30\end{array} & \begin{array}{l}\text { Requiere tratamiento } \\ \text { electivo }\end{array}\end{array}$

\begin{tabular}{lll}
\hline Maloclusión severa & $\begin{array}{l}\text { Resultado del IED } \\
\text { entre } 30-35\end{array}$ & $\begin{array}{l}\text { Requiere tratamiento } \\
\text { deseable por el } \\
\text { paciente }\end{array}$ \\
\hline $\begin{array}{l}\text { Maloclusión muy } \\
\text { severa }\end{array}$ & $\begin{array}{l}\text { Resultado mayor o } \\
\text { igual a 36 }\end{array}$ & $\begin{array}{l}\text { Requiere tratamiento } \\
\text { prioritario }\end{array}$ \\
\hline
\end{tabular}

*IED: Indice de Estética Dental 
Tabla 3: Prevalencia y necesidad de tratamiento ortodóncico, según IED.

\begin{tabular}{|c|c|c|c|c|c|c|c|c|}
\hline \multirow[b]{2}{*}{ Género } & \multicolumn{2}{|c|}{$\begin{array}{l}\text { Oclusión normal } \\
\text { IED }<25\end{array}$} & \multicolumn{2}{|c|}{$\begin{array}{l}\text { Maloclusión definida } \\
\text { IED } 26-30\end{array}$} & \multicolumn{2}{|c|}{$\begin{array}{l}\text { Maloclusión severa } \\
\text { IED31 - } 35\end{array}$} & \multicolumn{2}{|c|}{$\begin{array}{l}\text { Maloclusión discapacitante } \\
\text { IED> } 36\end{array}$} \\
\hline & $\mathrm{N}$ & $\%$ & $\mathrm{~N}$ & $\%$ & $N$ & $\%$ & $\mathrm{~N}$ & $\%$ \\
\hline Masculino & 10 & 10,3 & 5 & 5,2 & 6 & 6,2 & 24 & 24,7 \\
\hline Femenino & 7 & 7,2 & 11 & 11,3 & 10 & 10,3 & 24 & 24,7 \\
\hline Total & 17 & 17,5 & 6 & 16,5 & 16 & 16,5 & 48 & 49,5 \\
\hline $\begin{array}{l}\text { Necesidad } \\
\text { tratamiento }\end{array}$ & $\begin{array}{l}\text { No necesita } \\
\text { tratamiento }\end{array}$ & $\begin{array}{l}\text { Tratamiento } \\
\text { electivo }\end{array}$ & $\begin{array}{l}\text { Tratamiento } \\
\text { conveniente }\end{array}$ & $\begin{array}{l}\text { Tratamiento } \\
\text { prioritario }\end{array}$ & & & & \\
\hline
\end{tabular}

*IED: Indice de Estética Dental

Tabla 4: Pacientes según componentes del Índice de Estética Dental y sexo

\begin{tabular}{|c|c|c|c|c|}
\hline \multicolumn{5}{|c|}{ IED n= 97} \\
\hline \multicolumn{2}{|c|}{ Componente } & Hombre & Mujer & Total \\
\hline \multirow{2}{*}{$\begin{array}{l}\text { Diente perdido } \\
\text { anterior }\end{array}$} & 0 & 40 & 48 & 88 \\
\hline & 1-3 dientes & 5 & 4 & 9 \\
\hline \multirow{2}{*}{$\begin{array}{l}\text { Apiñamiento } \\
\text { sector incisal }\end{array}$} & 0 & 9 & 11 & 20 \\
\hline & $\begin{array}{c}1-2 \\
\text { segmentos }\end{array}$ & 36 & 41 & 77 \\
\hline \multirow{2}{*}{$\begin{array}{l}\text { Espaciamiento } \\
\text { sector incisal }\end{array}$} & 0 & 32 & 36 & 68 \\
\hline & $\begin{array}{c}1-2 \\
\text { segmentos }\end{array}$ & 13 & 16 & 29 \\
\hline \multirow{2}{*}{ Diastema } & 0 & 37 & 42 & 79 \\
\hline & $1-\geq 3 \mathrm{~mm}$ & 8 & 10 & 18 \\
\hline \multirow{2}{*}{$\begin{array}{l}\text { Irregularidad } \\
\text { anterior maxilar }\end{array}$} & 0 & 13 & 11 & 24 \\
\hline & $>1 \mathrm{~mm}$ & 32 & 41 & 73 \\
\hline \multirow{2}{*}{$\begin{array}{l}\text { Irregularidad } \\
\text { anterior } \\
\text { mandibular }\end{array}$} & 0 & 10 & 11 & 21 \\
\hline & $>1 \mathrm{~mm}$ & 35 & 41 & 76 \\
\hline \multirow{2}{*}{ Resalte maxilar } & $0-2 \mathrm{~mm}$ & 10 & 14 & 24 \\
\hline & $>2 \mathrm{~mm}$ & 35 & 38 & 73 \\
\hline \multirow{2}{*}{$\begin{array}{l}\text { Resalte } \\
\text { mandibular }\end{array}$} & 0 & 44 & 51 & 95 \\
\hline & $\geq 1 \mathrm{~mm}$ & 1 & 1 & 2 \\
\hline \multirow{2}{*}{$\begin{array}{l}\text { Mordida abierta } \\
\text { anterior }\end{array}$} & 0 & 45 & 49 & 94 \\
\hline & $\geq 1 \mathrm{~mm}$ & 0 & 3 & 3 \\
\hline \multirow{3}{*}{ Relación molar } & Normal & 13 & 17 & 30 \\
\hline & $\begin{array}{l}\text { Media } \\
\text { cúspide }\end{array}$ & 24 & 32 & 56 \\
\hline & $\begin{array}{l}\text { Cúspide } \\
\text { completa }\end{array}$ & 8 & 3 & 11 \\
\hline
\end{tabular}

*IED: Indice de Estética Dental (espaciamiento $\times 1)+($ diastema $\times 3)+($ máxima irregularidad maxilar anterior $\times 7)+($ máxima irregularidad mandibular anterior $\times 1)+($ Overjet maxilar $\times 2)+($ Overjet mandibular $\times 4)+($ mordida abierta anterior $\times 4)$

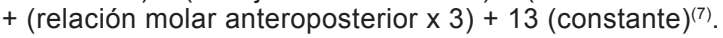

Una vez obtenido este valor numérico, se realizó la clasificación de maloclusión y necesidad de tratamiento ${ }^{(9)}$ (Tabla 2).

Finalmente, se realizó estadística descriptiva mediante una planilla excel 2016.

\section{RESULTADOS}

De los 97 pacientes examinados, 45 (46.3\%) fueron de sexo masculino y $52(53.7 \%)$ de sexo femenino. En el análisis de frecuencias se obtuvo un valor mínimo de puntaje del IED de 21 y un valor máximo de 89 (Tabla 3)

Un $17.52 \%$ de los adolescentes examinados obtuvo puntajes del IED $\leq 25$, indicativo de oclusión normal o maloclusión mínima, lo que determina que el tratamiento ortodóncico sea innecesario o poco necesario, un $16.49 \%$ obtuvo una puntuación entre 26 y 30 indicativo de maloclusión manifiesta y una necesidad de tratamiento optativa, un $16.49 \%$ obtuvo un puntaje entre 31 y 35 indicativo de maloclusión severa y tratamiento sumamente deseable y un $49.48 \%$ obtuvo una puntuación $\geq 36$ indicativo de maloclusión muy severa o discapacitante con una necesidad de tratamiento obligatoria (Tabla 3 ).

Del análisis de cada uno de los componentes del IED entre hombres y mujeres se obtuvieron los resultados contenidos en la Tabla 4.

\section{DISCUSIÓN}

Aplicando el IED en este estudio, se encontró una necesidad de tratamiento de $82.5 \%$, dada por las maloclusiones de tipo definida, severa y discapacitante.

El promedio del IED fue de un 36.2, es decir, dentro del rango muy severo. El alto promedio se explica debido a que el $49.48 \%$ de los pacientes examinados se encuentra en este rango que requiere tratamiento prioritario.

Por otro lado, $17.52 \%$ de la población estudiada no necesita tratamiento, ya que presentan una oclusión normal o maloclusión leve.

Estos resultados muestran una prevalencia de maloclusiones y su respectiva necesidad de tratamiento similar a lo encontrado por estudios realizados en Chile. Cartes-Velásquez et al.(10) encontró un $67.4 \%$ de maloclusiones. Otro estudio del año 2014(11) examinó 129 pacientes de 12 años y evidenció una necesidad de tratamiento ortodóncico mediato/inmediato en un $64.3 \%$ de los participantes. Sin embargo, al comparar solo el rango discapacitante o muy severo, este estudio presentó valores más elevados.

En comparación con investigaciones realizadas en otros países latinoamericanos, se encontraron índices de necesidad de tratamiento siempre menores, Colombia $67.9 \%^{(12)}$, Cuba $67.1 \%{ }^{(9)}$ y México $46.8 \%{ }^{(13)}$.

En Brasil(14), se reporta una prevalencia de maloclusiones del $31.3 \%$.

Estudios realizados en otros continentes muestran resultados aún menores, como España ${ }^{(15)}$ con un $41.4 \%$ de necesidad de tratamiento y un $9.9 \%$ para el nivel discapacitante. Por otro lado, estudios en África ${ }^{(16)}$ reportan índices más elevados con una necesidad del $47 \%$. 
Las pocas oportunidades de acceso a tratamiento preventivo y restaurador de caries, tanto en el sistema público como en el ámbito privado, puede conducir a la pérdida prematura de dientes temporales, lo que a su vez incide en la aparición de apiñamiento en la dentición permanente. Sumado a lo anterior, el aislamiento geográfico y la nula disponibilidad de tratamiento de especialidad de ortodoncia en la ciudad donde habitan los estudiantes, podrían dar cuenta de los altos índices de maloclusión moderada y severa encontrados en el presente estudio(1). Sin embargo, los resultados no se podrían explicar solo con los factores mencionados y sería recomendable realizar más estudios que pudiesen ahondar en el grado de influencia de otras causas.

En la Tabla 4, se observa la distribución de los componentes del IED, ordenados por sexo y sus respectivos porcentajes. La maloclusión más frecuente fue el apiñamiento en el sector incisal encontrada en un $79.4 \%$ de los pacientes, seguida por la irregularidad anterior de la mandíbula con un $78.4 \%$. Estos resultados, si bien son más altos que lo encontrado en estudios similares, son coincidentes en cuanto a ser los componentes con más altos porcentajes dentro de la muestra ${ }^{(12,17)}$.

Una revisión sistemática muestra la evidencia disponible respecto a los efectos negativos en la calidad de vida relacionada con la salud oral en el ámbito emocional y social en jóvenes con maloclusión ${ }^{(2)}$. Bittencourt et al. ${ }^{(14)}$ encontraron una asociación significativa entre el grado de maloclusión presentado y el bienestar emocional y social de los adolescentes, basado en un cuestionario de auto-percepción infantil en su versión brasileña. La preocupación por la estética dental es muy común en la sociedad actual, ya que tiene un efecto directo en la calidad de vida, relacionada principalmente con la aceptación social ${ }^{(2,14)}$.

De esta forma, un problema de salud altamente prevalente, con repercusiones de amplio impacto psicosocial y bajo el principio de equidad en el acceso a los servicios de salud, es un llamado a la acción para dar respuesta al menos al $49.5 \%$ de adolescentes que requieren tratamiento ortodóncico prioritario, convirtiendo a este estudio en un diagnóstico local inicial. Considerando una amplia lista de espera(1) y una oferta de servicios escasa, conviene aplicar criterios estandarizados para la priorización de los casos según su nivel de complejidad. El IED ha permitido priorizar a quienes necesitan tratamiento ${ }^{(18)}$, estableciendo una medición estandarizada validada por consenso entre ortodoncistas ${ }^{(4,5)}$, así como también se ha sugerido la autopercepción de necesidad de tratamiento entre adolescentes de Brasil(19).

Dada la alta prevalencia de adolescentes que requieren tratamiento ortodóncico prioritario, encontrada en este estudio desde un punto de vista normativo, convendría la consiguiente aplicación de encuestas con un enfoque socio-dental, el que puede ayudar a maximizar el uso de los recursos al diferenciar a quienes probablemente resultarían más beneficiados al recibir el tratamiento(20,21). Paralelamente, se hace necesario reevaluar a aquellos adolescentes en lista de espera para ortodoncia que no fueron parte de la población estudiada.

Entre las estrategias que se proponen para abordar la situación, se encuentra la visita del personal odontológico de especialidad al box dental del Centro de Salud local y consultorías presenciales o a distancia, además del aumento de la oferta de servicios de odontología general y ortodoncia tradicionales.

\section{CONCLUSIÓN}

En la ciudad de Diego de Almagro existe una mayor necesidad de tratamiento ortodóncico según el IED en comparación con estudios nacionales e internacionales, lo que justifica el aumento de la cobertura de estrategias de salud bucal adaptadas a la realidad local, que abarque desde la prevención de patologías orales en edades tempranas, hasta el tratamiento para dar respuesta a las maloclusiones ya establecidas.

\section{RELEVANCIA CLÍNICA}

El Índice de Estética Dental es una herramienta simple y que puede ser replicada con relativa facilidad por odontólogos generales. Este estudio da a conocer la existencia de una alta necesidad de tratamiento ortodóncico en la población adolescente de 12 años que además presentan una maloclusión severa o muy severa. Esta información espera ser un aporte en la toma de decisiones gubernamentales en el ámbito de la salud oral.

\section{CONFLICTO DE INTERÉS}

No existen conflictos de interés

\section{FUENTE DE FINANCIAMIENTO}

Autofinanciado

\section{AGRADECIMIENTO}

A nuestro querido Hospital Florencio Vargas Díaz, sus funcionarios y toda la comunidad de Pueblo Hundido por haber hecho posible esta investigación. 


\section{Bibliografía}

1. Ministerio de Salud. Lista de Espera GES y NO GES. ORD N ${ }^{\circ} 496 / 2019$ (31 de enero de 2019) [Internet]. 2018 [citado 17 octubre 2019]. Disponible en: https://www. minsal.cl/wp-content/uploads/2019/05/Glosa-6-Dic-2018-Finalfallecidos-ords.pdf

2. Dimberg L, Arnrup K, Bondemark L. The impact of malocclusion on the quality of life among children and adolescents: A systematic review of quantitative studies. Eur J Orthod. 2015;37(3):238-47.

3. Bauman JM, Souza JGS, Bauman CD, Flório FM. Aspectos sociodemográficos relacionados à gravidade da maloclusão em crianças Brasileiras de 12 anos. Cien Saude Colet. 2018;23(3):723-32.

4. Costa R, Abreu MH, Magalhães CS, Moreira AN. Validity of two occlusal indices for determining orthodontic treatment needs of patients treated in a public university in Belo Horizonte, Minas Gerais State, Brazil. Cad Saude Publica. 2011;27(3):581-90. 5. Beglin FM, Firestone AR, Vig KW, Beck FM, Kuthy RA, Wade D. A comparison of the reliability and validity of 3 occlusal indexes of orthodontic treatment need. Am J Orthod Dentofacial Orthop. 2001;120(3):240-6.

6. Organización Mundial de la Salud. Encuestas de salud bucodental. Ginebra: OMS; 1997.

7. Jenny J, Cons N. Guide lines for using the DAI. A supplement to DAI the Dental Aesthetic Index. lowa City; University of lowa. 1988.

8. Marmot M, Friel S, Bell R, Houweling T, Taylor S. Closing the gap in a generation: health equity through action on the social determinants of health. Lancet 2008;372(9650):1661-9

9. Alemán M, Martínez I, Pérez A. Necesidad de tratamiento ortodóncico en escolares. Aplicación del índice DAI. Rev Med Electrón. 2011;33(4):441-7.

10. Cartes-Velásquez R, Araya $E$, Valdés $C$. Maloclusiones y su impacto psicosocial en estudiantes de un liceo intercultural. Int J Odontostomat. 2010;4(1):65-70.

11. Pérez MA, Neira A, Alfaro J, Aguilera J, Alvear P, Fierro Monti C. Necesidad de tratamiento ortodóncico según el índice de estética dental en adolescentes de 12 años, Chile. Rev Fac Odontol Univ Antioq. 2014;26(1):33-43.

12. Mafla AC, Barrera DA, Muñoz GM. Malocclusion and orthodontic treatment need in adolescents from Pasto, Colombia. Rev Fac Odontol Univ Antioq. 2011;22(2):173-85.
13. Pérez V, García G, Cárdenas A, Carrasco R, Castro C, Lezama G, et al. Índice estética dental (DAI) y necesidad de tratamiento ortodóncico en escolares, verano 2007.Rev Oral. 2008;9(29):472-5.

14. Bittencourt JM, Martins LP, Bendo CB, Vale MP, Paiva SM. Negative effect of malocclusion on the emotional and social well-being of Brazilian adolescents: a population-based study. Eur J Orthod. 2017;39(6):628-33.

15. Baca-García A, Bravo M, Baca P, Baca A, Junco P. Malocclusions and orthodontic treatment needs in a group of Spanish adolescents using the Dental Aesthetic Index. Int Dent J. 2004;54(3):138-42.

16. Muasya MK, Ng'Ang'a M, Opinya GN, Macigo FG. Malocclusion and orthodontic treatment need among 12-15-year-old children in Nairobi. East Afr Med J. 2012;89(2):39-44

17. Mai $\mathrm{AH}$, Fernández YR, Delgado $\mathrm{CL}$, Coutín MG. Necesidad de tratamiento ortodóncico. Politécnico Antonio Guiteras. Plaza. 2012. Rev habanera cienc médi. 2014;13(6):855-61. Available from: http://scielo.sld.cu/scielo.php?script=sci_ arttext\&pid=S1729-519X2014000600006

18. Maumela PM, Hlongwa P. Application of the dental aesthetic index in the prioritization of orthodontic service needs. SADJ. 2012;67(7):380-3

19. Silva LF, Thomaz EB, Freitas HV, Ribeiro CC, Pereira AL, Alves CM. Selfperceived need for dental treatment and related factors. A cross-sectional population-based study. Braz Oral Res. 2016;30(1):e55. Available from http://www. scielo.br/scielo.php?script=sci_arttext\&pid=S1806-83242016000100259\&lng=en

20. Gherunpong S, Tsakos G, Sheiham A. A socio-dental approach to assessing children's orthodontic needs. Eur J Orthod. 2006;28(4):393-9.

21. Herkrath FJ, Rebelo MA, Herkrath AP, Vettore MV. Comparison of normative methods and the sociodental approach to assessing orthodontic treatment needs in 12-year-old schoolchildren. Oral Health Prev Dent. 2013;11(3):211-20. 